Annals of Pure and Applied Mathematics

Vol. 13, No. 2, 2017, 297-304

ISSN: 2279-087X (P), 2279-0888(online)

Published on 19 May 2017

www.researchmathsci.org

DOI: http://dx.doi.org/10.22457/apam.v13n2al6

Annals of

Pure and Applied

Mathematics

\title{
On a Ramsey Problem Involving Quadrilaterals
}

\author{
C.J.Jayawardene and T.U.Hewage
}

Department of Mathematics, University of Colombo, Colombo 3, Sri Lanka

Corresponding author. Email: c_jayawardene@yahoo.com

Received 26 April 2017; accepted 11 May 2017

\begin{abstract}
Let $j \geq 3$. Given any two coloring (consisting of say red and blue colors) of the edges of a complete graph $K_{j \times s}$, we say that $K_{j \times s} \rightarrow\left(C_{4}, G\right)$, if there exists a copy of a red $C_{4}$ or a copy of blue $G$ in it. Let $m_{j}\left(C_{4}, G\right)$ denote the smallest positive integer $s$ such that $K_{j \times s} \rightarrow\left(C_{4}, G\right)$. This paper deals with finding the exact values $m_{j}\left(C_{4}, G\right)$ for all possible proper subgraphs $G$ of $K_{4}$.
\end{abstract}

Keywords: Ramsey theory, multipartite Ramsey numbers

AMS Mathematics Subject Classification (2010): 05C55, 05D10

\section{Introduction}

All graphs listed in this paper are graphs without loops or parallel edges. Let $G, H$ and $K$ represent three graphs. Given any two coloring (consisting of say red and blue colors) of the edges of a graph $G$, we say that $G \rightarrow(H, K)$ if there exists a red copy of $H$ in $G$ or a blue copy of $K$ in $G$. The Ramsey number $r(H, K)$ is defined as the smallest positive integer $t$ such that $K_{t} \rightarrow(H, K)$. The diagonal classical Ramsey number $r(n, n)$ is defined as the smallest positive integer $t$ such that $K_{t} \rightarrow\left(K_{n}, K_{n}\right)$. In the last four decades most of the Ramsey numbers $R(H, K)$ have been studied in detail for $|V(H)|<7$ and $|V(K)|<7$ (see [6]). The size Ramsey multipartite number $m_{j}(H, K)$ is defined as the smallest natural number $t$ such that $K_{j \times t} \rightarrow(H, K)$ (see [1,3,5,7]). In this paper we concentrate on determining multipartite Ramsey numbers $m_{j}\left(C_{4}, G\right)$ for all possible proper subgraphs $G$ of $K_{4}$.

\section{Notation}

The vertices of $K_{j \times s}$ are labeled as $\left\{v_{k, i} \mid 1 \leq i \leq s, 1 \leq k \leq j\right\}$, with the $m^{\text {th }}$ partite set consisting of $\left\{v_{m, i} \mid 1 \leq i \leq s\right\}$. It is worth noting that all values of $m_{j}\left(C_{4}, P_{4}\right)$ and $m_{j}\left(C_{4}, C_{3}\right)$ (see [4])are currently known. Also all values of $m_{j}\left(C_{4}, K_{1,3}+x\right)$ are known as $m_{j}\left(C_{4}, C_{3}\right)=$ $m_{j}\left(C_{4}, K_{1,3}+x\right)$ for any integer $j$.

3. Some useful lemmas on connected proper subgraphs of $\boldsymbol{K}_{\mathbf{4}}$

Theorem 1. If $j \geq 3$, then

$$
m_{j}\left(C_{4}, C_{4}\right)= \begin{cases}1 & j \geq 6 \\ 2 & j=\{4,5\} \\ 3 & j=3\end{cases}
$$




\section{C.J.Jayawardene and T.U.Hewage}

Proof. If $j \geq 6$ (see [2]), since $r\left(C_{4}, C_{4}\right)=6$ we get $m_{j}\left(C_{4}, C_{4}\right)=1$. So we are left with the cases $j=3, j=4$ and $j=5$. If $j=4$ or $j=5$, consider the coloring of $K_{j \times 1}=H_{R} \oplus H_{B}$, generated by $H_{R}=C_{5}$ and $H_{R}=C_{3}$ respectively. Then, $K_{j \times 1}$ has no red $C_{4}$ or a blue $C_{4}$. Therefore, we obtain that $m_{4}\left(C_{4}, C_{4}\right) \geq 2$ and $m_{5}\left(C_{4}, C_{4}\right) \geq 2$.

In order to show, $m_{5}\left(C_{4}, C_{4}\right) \leq 2$ and $m_{4}\left(C_{4}, C_{4}\right) \leq 2$, first note that it suffices only to show that $m_{4}\left(C_{4}, C_{4}\right) \leq 2$. Consider any red/blue coloring given by $K_{4 \times 2}=H_{R} \oplus H_{B}$, such that $H_{R}$ contains no red $C_{4}$ and $H_{B}$ contains no blue $C_{4}$.

In the first possibility that $H_{R}$ is a regular graph of order 3 , we get from the above remark that $H_{R}$ must contain a red $C_{3}$. Without loss of generality assume that this red 3 cycle is incident to the first three partite sets and consists of say $v_{1,1}, v_{2,1}, v_{3,1}$. Then both $v_{4,1}$ and $v_{4,2}$ have to be adjacent to two verticesof $v_{1,1}, v_{2,1}, v_{3,1}$ in blue in order to avoid a red $C_{4}$. Without loss of generality assume $v_{4,1}$ and $v_{4,2}$ are adjacent $v_{3,1}, v_{2,1}$ and $v_{3,1}, v_{1,1}$ respectively. But then as $\operatorname{deg}_{R}\left(v_{1,1}\right)=3,\left(v_{1,1}, v_{2,2}\right)$ and $\left(v_{1,1}, v_{3,2}\right)$ will have to be blue edges. This is illustrated in the following figure.

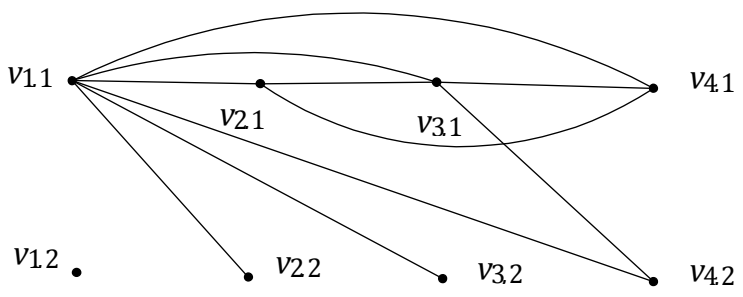

Figure 1: In the first possibility, the derived red/blue graphs

As there is no blue $C_{4},\left(v_{2,2}, v_{3,1}\right)$ and $\left(v_{4,2}, v_{2,1}\right)$ have to be red edges. Next as there is no red $C_{4},\left(v_{2,2}, v_{4,1}\right)$ has to be a blue edge. But then if we consider the edge $\left(v_{2,2}, v_{4,2}\right)$ we see that it can be neither a red edge or a blue edge as it will give rise to a red $C_{4}$ or a blue $C_{4}$, a contradiction.In the second possibility that $H_{R}$ is not a regular graph of order 3 , by symmetry we can assume that red degree of a vertex (say $v_{1,2}$ ) is greater than or equal to 4 and without loss of generality $v_{1,2}$ is connected in red to $v_{2,1}, v_{2,2}, v_{3,1}$ and $v_{4,1}$ as illustrated in the following figure (note if $v_{1,2}$ is adjacent to $v_{2,1}, v_{2,2}, v_{3,1}$ and $v_{3,2}$, it would clearly force a monochromic.

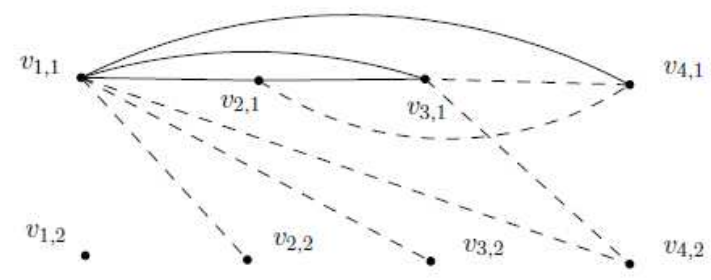

Figure 2: In the first possibility, the derived red/blue graphs 


\section{On a Ramsey Problem Involving Quadrilaterals}

If $v_{1,1}$ is connected in red to two vertices of $N_{R}\left(v_{1,2}\right)$ will result in a red $C_{4}$. Therefore, without loss of generality get either $v_{1,1}$ to connected in blue to $v_{2,1}, v_{3,1}, v_{4,1}$ or else $v_{1,1}$ to connected in blue to $v_{2,1}, v_{2,2}, v_{3,1}$. In the first scenario, as the induced subgraph of $v_{2,1}, v_{3,1}$, $v_{4,1}$ will contains 2 red edges or 2 blue edges and these two edges will be contained in a $C_{4}$ of that same color, a contradiction. In the second scenario, $v_{4,2}$ will be adjacent to two vertices of $v_{2,1}, v_{2,2}, v_{3,1}$ in some color and then this would force these two vertices to be contained in a monochromatic $C_{4}$ in that same color, a contradiction.

If $j=3$, consider the coloring of $K_{j \times 2}=H_{R} \oplus H_{B}$, generated by $H_{R}=2 K_{3}$. Then, $K_{j \times 2}$ has no red $C_{4}$ or a blue $C_{4}$. Therefore, we obtain that $m_{3}\left(C_{4}, C_{4}\right) \geq 3$.

To show, $m_{3}\left(C_{4}, C_{4}\right) \leq 3$. Consider any red/blue coloring given by $K_{3 \times 3}=H_{R} \oplus H_{B}$, such that $H_{R}$ contains no red $C_{4}$ and $H_{B}$ contains no blue $C_{4}$. By handshaking lemma all vertices cannot have red degree 3 .

Therefore, without loss of generality, using symmetry, we may assume that $v_{1,1}$ is adjacent to at least 4 vertices in red. Let $V$ be any subset of size 4 of $N_{R}\left(v_{1,1}\right)$. In order to avoid a red $C_{4}$, both $v_{1,2}$ and $v_{1,3}$ must be adjacent to at least three vertices of $V$ in blue. This will result in a blue $C_{4}$ containing $v_{1,2}$ and $v_{1,3}$ contrary to our assumption. Therefore, we could conclude that $m_{3}\left(C_{4}, C_{4}\right)=3$.

\section{Theorem 2.}

$$
m_{j}\left(C_{4}, B_{2}\right)= \begin{cases}1 & j \geq 7 \\ 2 & j=\{5,6\} \\ 3 & j=4 \\ 4 & j=3\end{cases}
$$

Proof. Clearly $m_{j}\left(C_{4}, B_{2}\right)=1$ if $j \geq 7$, since $r\left(C_{4}, B_{2}\right)=7$ (see [2]).

Let $j \in\{5,6\}$. Consider the coloring of $K_{j \times 1}=H_{R} \oplus H_{B}$, generated by $H_{R}=C_{5}$ and $H_{R}=2 K_{3}$ when $j=5$ and $j=6$ respectively. Then, $K_{j \times 1}$ has no red $C_{4}$ or a blue $B_{2}$. Therefore, we obtain that $m_{5}\left(C_{4}, B_{2}\right) \geq 2$ and $m_{6}\left(C_{4}, B_{2}\right) \geq 2$. Next we have to show $m_{5}\left(C_{4}, B_{2}\right) \leq 2$. For this consider any coloring consisting of (red, blue) given by $K_{5 \times 2}=H_{R} \oplus H_{B}$, such that $H_{R}$ contains no red $C_{4}$ and $H_{B}$ contains no red $B_{2}$. Then since $m_{5}\left(C_{4}, C_{3}\right)=2$, without loss of generality we may assume that $\left(v_{1,1}, v_{2,1}, v_{3,1}\right)$ is a blue cycle. Define $T=\left\{v_{4,1}, v_{4,2}, v_{5,1}, v_{5,2}\right\}$ and $S=\left\{v_{1,1}, v_{2,1}, v_{3,1}\right\}$. Then, if any vertex of $T$ is adjacent to two vertices of $S$ in blue, it will result blue $B_{2}$, contrary to our assumption. Therefore, we will be left with the option every vertex of $T$ is adjacent to at least two vertices of $S$ in red. But then as $|S|=3$ there will be twovertices of $T$ adjacent in red to the same pair of vertices in $S$. This will result in a red $C_{4}$, a contradiction. From this we can conclude that $m_{j}\left(C_{4}, B_{2}\right) \leq 2$ if $j \in\{5,6\}$. That is, $m_{j}\left(C_{4}, B_{2}\right)=2$ if $j \in\{5,6\}$. We are left with the following two cases, namely $j=$ 4 (case 1 ) and $j=3$ (case 2).

Case 1: $j=4$

Consider the coloring of $K_{4 \times 2}=H_{R} \oplus H_{B}$, generated by $H_{R}$ illustrated in the following figure. 


\section{C.J.Jayawardene and T.U.Hewage}

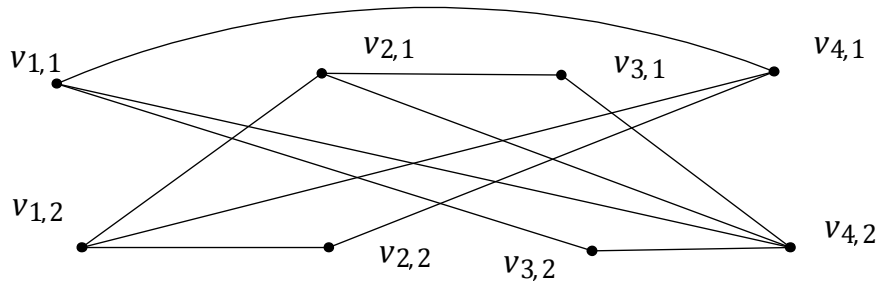

Figure 3: Case 1

Then $H_{R}$ will contain three disjoint triangles except for two triangles containing a common vertex. Thus the red-blue coloring generated by the figure will be such that $H_{R}$ contains no red $C_{4}$ and $H_{B}$ contains no blue $B_{2}$. Therefore, we will get $m_{4}\left(C_{4}, B_{2}\right) \geq 3$. To show that $m_{3}\left(C_{4}, B_{2}\right) \leq 3$ consider any coloring consisting of (red , blue) given by $K_{3 \times 4}=H_{R}$ $\bigoplus H_{B}$, such that $H_{R}$ contains no red $C_{4}$ and $H_{B}$ contains no blue $B_{2}$. Since $m_{4}\left(C_{4}, C_{3}\right)=2$ without loss of generality, we get that, $v_{1,1} v_{2,1} v_{3,1} v_{1,1}$ is a blue cycle. Define $S=$ $\left\{v_{1,1}, v_{2,1}, v_{3,1}\right\}$ and $T=\left\{v_{4,1}, v_{4,2}, v_{4,3}\right\}$. If any vertex of $T$ has adjacent in blue to 2 vertices of $S$ we will get a blue $B_{2}$ and if any two vertex of $T$ has adjacent in red to the same 2 vertices of $S$ we will get a red $C_{4}$. Therefore, we may assume that $v_{4,1}$ is adjacent in blue to $v_{1,1}$ and in red to $v_{2,1}$ and $v_{3,1} ; v_{4,2}$ is adjacent in blue to $v_{2,1}$ and in red to $v_{1,1}$ and $v_{3,1} ; v_{4,3}$ is adjacent in blue to $v_{3,1}$ and in red to $v_{1,1}$ and $v_{2,1}$.

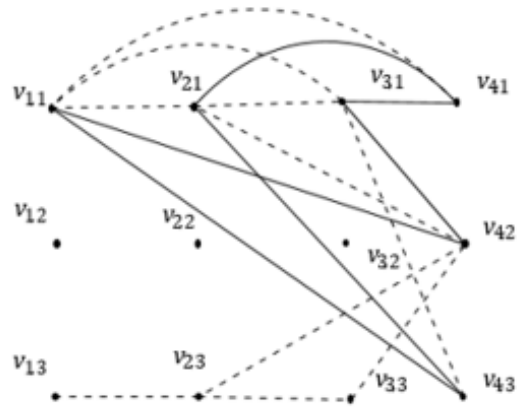

Figure 4: The first scenario

Also in the remaining 6 vertices (in $S^{c} \cap T^{c}$ ) must contain a blue $P_{3}$ as $m_{3}\left(C_{4}, P_{3}\right)=2$. Thus, without loss of generality we may assume that $v_{1,3} v_{2,3} v_{3,3}$ is a blue $P_{3}$. In order to avoid a red $C_{4}$, all three vertices of $\left\{v_{1,3}, v_{2,3}, v_{3,3}\right\}$ must be adjacent in blue to at least two vertices of $T$. Thus, without loss of generality this gives rise to two possible scenarios illustrated in Figure 5 and Figure 6 respectively.

In the first scenario, in order to avoid a blue $B_{2}$ both $\left(v_{1,3}, v_{4,2}\right)$ and $\left(v_{1,3}, v_{3,3}\right)$ must be a red edges. Then in order to avoid a red $C_{4},\left(v_{1,1}, v_{3,3}\right)$ must be a blue edge; in order to avoid a blue $B_{2},\left(v_{3,3}, v_{2,1}\right)$ and $\left(v_{1,1}, v_{2,3}\right)$ must be red edges; in order to avoid a red $C_{4},\left(v_{3,1}, v_{2,3}\right),\left(v_{1,3}, v_{4,3}\right)$ and $\left(v_{1,3}, v_{4,1}\right)$ must be a blue edges. But then in order to avoid a blue $B_{2},\left(v_{2,3}, v_{4,3}\right)$ must be red. In order to avoid a red $C_{4},\left(v_{2,3}, v_{4,1}\right)$ must be a blue edge. 
On a Ramsey Problem Involving Quadrilaterals

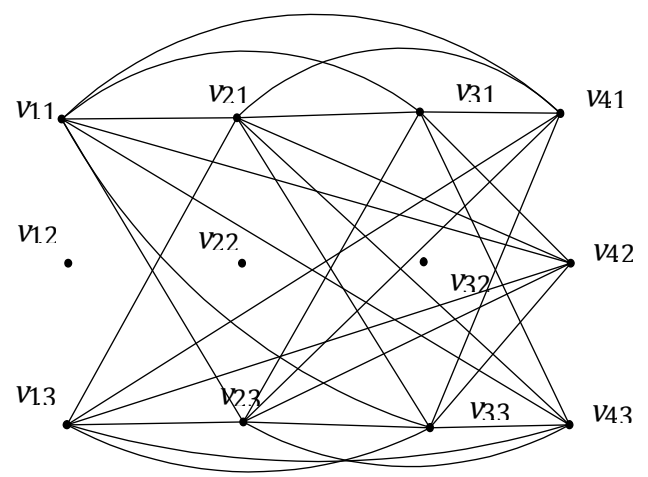

Figure 5: The final graph generated by the first scenario

In order to avoid a blue $B_{2},\left(v_{3,3}, v_{4,1}\right)$ must be a red edge and in order to avoid a red $C_{4}$, $\left(v_{1,3}, v_{2,1}\right)$ must be a blue edge. The red/blue graph generated is illustrated in the above figure.

But then if $\left(v_{1,3}, v_{3,1}\right)$ is red we get a red $C_{4}$ and if is blue we get a blue we get a $B_{2}$, a contradiction.

In the second scenario, we may assume that $v_{4,1}$ is adjacent in blue to $v_{2,3}$ and $v_{3,3}$; $v_{4,2}$ is adjacent in blue to $v_{1,3}$ and $v_{3,3} ; v_{4,3}$ is adjacent in blue to $v_{1,3}$ and $v_{3,3}$. Next in order to avoid a blue $B_{2},\left(v_{3,3}, v_{4,3}\right),\left(v_{2,3}, v_{4,2}\right),\left(v_{1,3}, v_{4,1}\right)$ and $\left(v_{2,3}, v_{3,1}\right)$ must be a red edges. Then in order to avoid a red $C_{4},\left(v_{1,1}, v_{2,3}\right)$ must be a blue edge; in order to avoid a blue $B_{2}$, $\left(v_{3,3}, v_{1,1}\right)$ must be a red edge.In order to avoid a red $C_{4},\left(v_{2,1}, v_{3,3}\right)$ must be a blue edge; in order to avoid a blue $B_{2},\left(v_{1,3}, v_{2,1}\right)$ must be a red edge; and in order to avoid a red $C_{4}$, $\left(v_{1,3}, v_{3,1}\right)$ must be a blue edge. But then the vertices in $\left\{v_{1,3}, v_{3,1}, v_{4,3}, v_{2,3}\right\}$ will induce a blue $B_{2}$, a contradiction.

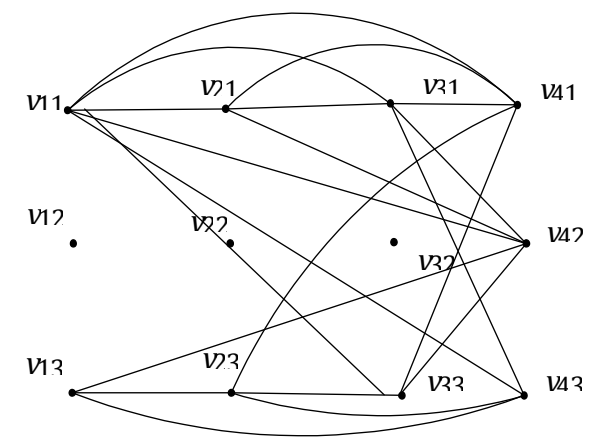

Figure 6: The second scenario

Case 2: $j=3$

Consider the coloring of $K_{3 \times 4}=H_{R} \oplus H_{B}$, generated by $H_{R}$ and $H_{B}$ illustrated in the following figure. The red blue coloring generated by the following figure will be such that $H_{R}$ contains no red $C_{4}$ and $H_{B}$ contains no red $B_{2}$. Therefore, we will get $m_{3}\left(C_{4}, B_{2}\right) \geq 4$. 

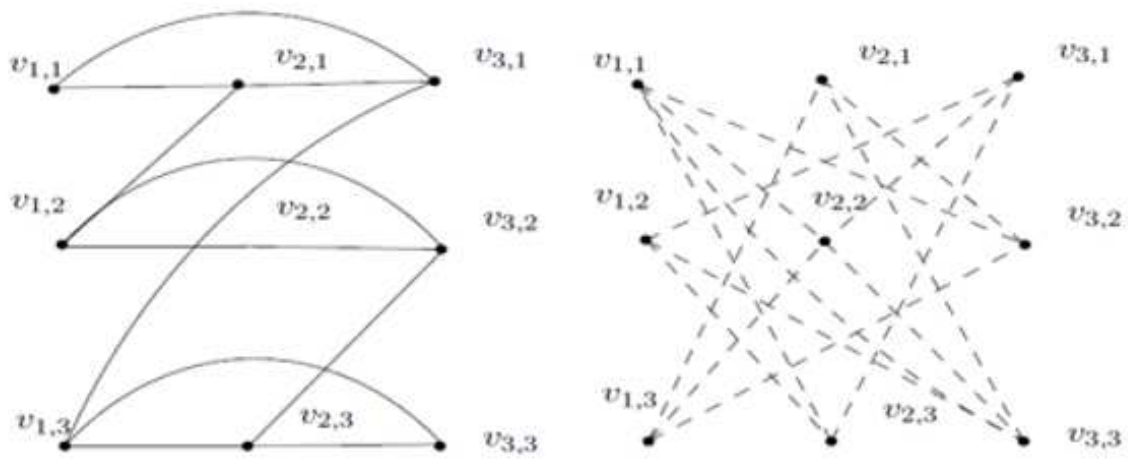

Figure 7: Case 2

To show that $m_{3}\left(C_{4}, B_{2}\right) \leq 4$, consider any coloring consisting of (red,blue) given by $K_{3 \times 4}$ $=H_{R} \oplus H_{B}$ such that there is no red $C_{4}$ in $H_{R}$ or a red $B_{2}$ in $H_{B}$. Subject to these conditions will first show the following three claims.

Notation: Let $1 \leq i, j \leq 4$. A vertex $v \in K_{3 \times 4}$ having blue degree $i+j$ is said to consists of a blue $(i, j)$ split if $v$ is adjacent in blue to $i$ vertices of one particle set and $j$ vertices of the other partite set.

Claim 1: All vertices of $K_{3 \times 4}$ have blue degree at most five.

Proof. Suppose $v_{1,4}$ has blue degree at least 6 . Without loss of generality, there are two possibilities. Then one of the following two scenarios must be true. The first $v_{1,4}$ is adjacent in blue to all vertices of $S=\left\{v_{2,1}, v_{2,2}, v_{2,3}, v_{2,4}, v_{3,3}, v_{3,4}\right\}$ and the second $v_{1,1}$ is adjacent in blue to all vertices of $S=\left\{v_{2,1}, v_{2,2}, v_{2,3}, v_{3,1}, v_{3,2}, v_{3,3}\right\}$.

In the first scenario to avoid a blue $B_{2}$, both $v_{3,3}$ and $v_{3,4}$ will have to be adjacent in red to at least three vertices of the second partite set. This will result in a red $C_{4}$ containing $v_{3,3}$ and $v_{3,4}$, a contradiction. Hence the claim follows.

In the second scenario, In order to avoid a blue $B_{2}$ all edges between $\left\{v_{2,1}, v_{2,2}, v_{2,3}\right\}$ and $\left\{v_{3,1}, v_{3,2}, v_{3,3}\right\}$. Next, applying $m_{3}\left(C_{4}, C_{3}\right)=3$ to $K_{3 \times 3}$ consisting of the first three elements of the three partite sets, we obtain a blue $B_{2}$ containing $v_{1,4}$. Hence the claim follows.

Claim 2: $K_{3 \times 4}$ has at least one vertex of blue degree five.

Proof. Applying $m_{3}\left(C_{4}, C_{3}\right)=3$ to $K_{3 \times 3}$ consisting of the first three elements of the three partite sets, without loss of generality we obtain a blue $C_{3}$ containing $S=\left\{v_{1,1}, v_{2,1}, v_{3,1}\right\}$. Next, as there is no blue $B_{2}$, each vertex outside $S$ will have to be adjacent in red to at least one vertex of $S$. Thus by pigeon-hole principle at least one vertex must have degree greater than 5 . Thus by Claim 1, we can conclude that $S$ has at least one vertex of blue degree five as required.

Claim 3: $K_{3 \times 4}$ has at least one vertex of blue $(3,2)$ split.

Proof. Suppose that the claim is false. Let $v_{1,4}$ be a vertex having a blue $(4,1)$ split. In particular, suppose that $v_{1,4}$ is adjacent in blue to all vertices of 


\section{On a Ramsey Problem Involving Quadrilaterals}

$S=\left\{v_{2,1}, v_{2,2}, v_{2,3}, v_{2,4}, v_{3,4}\right\}$ and adjacent in red to all vertices of $T=\left\{v_{3,1}, v_{3,2}, v_{3,3}\right\}$. Since there is no blue $B_{2}$ without loss of generality we may assume that $v_{3,4}$ adjacent in red to all vertices of $T=\left\{v_{2,1}, v_{2,2}, v_{2,3}\right\}$. Next we deal with 2 possible cases.

Case a: $v_{3,4}$ is adjacent in blue to at least one vertex of $\left\{v_{1,1}, v_{1,2}, v_{1,3}\right\}$ (say $v_{1,3}$ ). Then, since there is no red $C_{4}$ and by Claim 1 , we would get that $v_{1,3}$ will be a blue $(3,2)$ split.

Case b: $v_{3,4}$ is adjacent in red to all three vertices of $\left\{v_{1,1}, v_{1,2}, v_{1,3}\right\}$. Then, since there is no red $C_{4}$ and by Claim 1 , we would get that $v_{2,4}$ will be a blue $(3,2)$ split.

Now let us try to complete the proof of $j=3$ case. According to lemma 3, $v_{1,4}$ be a vertex having a blue $(3,2)$ split.

In particular, suppose that $v_{1,4}$ is adjacent in blue to all vertices of $S=\left\{v_{2,2}, v_{2,3}, v_{2,4}, v_{3,3}, v_{3,4}\right\}$ and adjacent in red to all vertices of $T=\left\{v_{2,1}, v_{3,1}, v_{3,2}\right\}$.

Since there is no blue $B_{2}$ or a red $C_{4}$, without loss of generality we may assume that $v_{3,4}$ adjacent in red to $v_{2,3}, v_{2,4}$ and that $v_{3,3}$ adjacent in red to $v_{2,2}, v_{2,3}$. Next as there is no red $C_{4}$, we would get that $\left(v_{2,4}, v_{3,3}\right)$ and $\left(v_{2,2}, v_{3,4}\right)$ are blue edges. This is illustrated in the following figure.

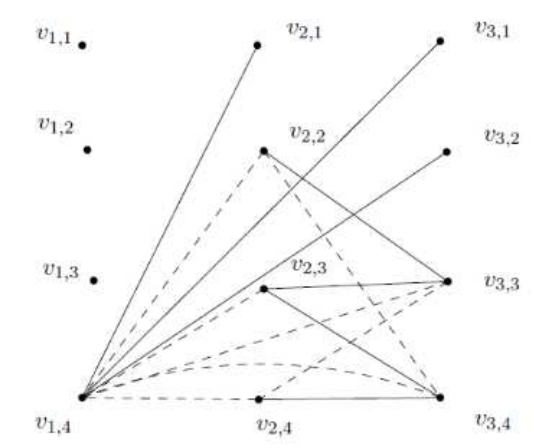

Figure 8: The generated graph for both cases 1 and 2, when $j=3$

Next we get that as in claim 3, there are two possible cases to consider.

Case 2.1: $\left(v_{2,3}, v_{1,3}\right)$ is red. First in order to avoid a red $C_{4}$ both $\left(v_{1,3}, v_{2,2}\right)$ and $\left(v_{1,3}, v_{2,4}\right)$ will have to be blue. Next in order to avoid a blue $B_{2}$ both $\left(v_{1,3}, v_{3,3}\right)$ and $\left(v_{1,3}, v_{3,4}\right)$ will have to be red. But this would give us $v_{1,3} v_{3,4} v_{2,3} v_{3,3} v_{1,3}$ is a red $C_{4}$.

Case 2.2: $v_{2,3}$ is adjacent in blue to all three vertices of $\left\{v_{1,1}, v_{1,2}, v_{1,3}\right\}$. Next, as there is no red $C_{4}, v_{2,3}$ will be adjacent to at least one vertex of $\left\{v_{3,1}, v_{3,2}\right\}$ in blue. Without loss of generality assume $\left(v_{2,3}, v_{3,2}\right)$ is blue. Then, by Claim $1,\left(v_{2,3}, v_{3,1}\right)$ will be red.Next, as there is no red $C_{4},\left(v_{2,2}, v_{3,1}\right)$ and $\left(v_{2,4}, v_{3,1}\right)$ will have to be blue.

In order to avoid a blue $B_{2}$ the vertex $v_{3,2}$ must be adjacent to two vertices of $\left\{v_{1,1}, v_{1,2}, v_{1,3}\right\}$ in red. Without loss of generality assume that $\left(v_{3,2}, v_{1,2}\right)$ and $\left(v_{3,2}, v_{1,3}\right)$ are red. But then in order to avoid a red $C_{4},\left(v_{3,1}, v_{1,2}\right)$ and $\left(v_{3,1}, v_{1,3}\right)$ are blue. Consider four vertices, $v_{3,1}$ is adjacent to in blue, given by $W=\left\{v_{1,2}, v_{1,3}, v_{2,2}, v_{2,4}\right\}$. In order to avoid a blue $B_{2}$ there can be at most one blue edge among them. That is there are three red edges in the subgraph induced by $W$. Exhaustive search will show that in each of the possibilities either $v_{1,2} v_{3,2} v_{1,3} v_{2,2} v_{1,2}$ or $v_{1,2} v_{3,2} v_{1,3} v_{2,4} v_{1,2}$ will be a red $C_{4}$, a contradiction. 


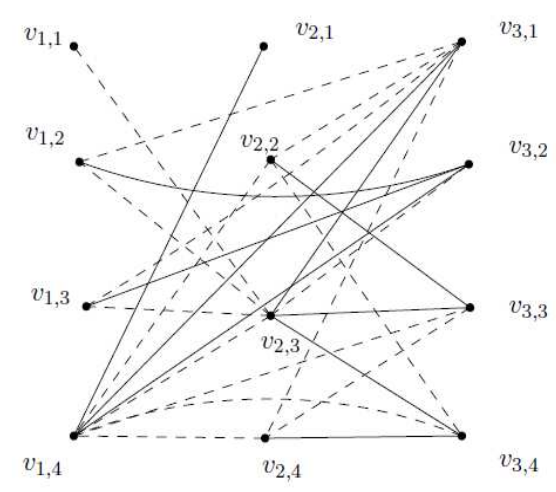

Figure 9: The final graph of case 2, when $j=3$

\section{Disconnected graphs up to 4 vertices}

It is worth noting that if $G$ and $H$ are two graphs with at most 4 vertices satisfying $G=H$ $\cup K_{1}$. Then clearly, $m_{j}\left(C_{4}, G\right)=m_{j}\left(C_{4}, H\right)$ for $j \geq 4$. In the case of $j=3$, as $m_{3}\left(C_{4}, H\right)>1$ for all connected graphs $H$ up to 3 vertices we $m_{3}\left(C_{4}, G\right)=m_{3}\left(C_{4}, H\right)$. Therefore, by this remark we are left are left only to consider $m_{j}\left(C_{4}, 2 K_{2}\right)$. However, this follows directly from $m_{j}\left(C_{4}, P_{4}\right)$ as $m_{j}\left(C_{4}, 2 K_{2}\right) \leq m_{j}\left(C_{4}, P_{4}\right)$ for any integer $j$.

\section{REFERENCES}

1. A.P.Burgerand J.H.van Vuuren, Ramsey numbers in complete balanced multipartite graphs. Part II: Size Numbers, Discrete Math., 283 (2004) 45-49.

2. V.Chv'atal and F.Harary, Generalized Ramsey theory for graphs, III. Small off diagonal numbers, Pacific Journal of Mathematics., 41-2 (1972) 335-345.

3. M.Christou, S.Iliopoulos and M. Miller, Bipartite Ramsey numbers involving stars, stripes and trees, Electronic Journal of Graph Theory and Applications, 1(2) (2013) 89-99.

4. C.J.Jayawardene and L.Samerasekara, Size multipartie Ramsey numbers for $C_{3}$ versus all graphs $G$ up to 4 vertices, Annals of Pure and Applied Mathematics, 13(1) (2017) 9-26.

5. C.J.Jayawardene and L.Samerasekara, Size multipartie Ramsey numbers for $K_{4}-e$ versus for all graphs up to 4 vertices, National Science Foundation, 45(1) (2017) 6772.

6. V.Kavitha and R.Govindarajan, A study on Ramsey numbers and its bounds, Annals of Pure and Applied Mathematics, 8(2) (2014) 227-236.

7. T.Pathinathan and J.Jesintha Rosline, Matrix representation of double layered fuzzy graph and its properties, Annals of Pure and Applied Mathematics, 8(2) (2014) 5158. 